Reprod. Nutr. Dévelop., 1986, 26 (1 B), 205-226.

\title{
Le métabolisme hépatique des glucides et des lipides chez les ruminants : principales interactions durant la gestation et la lactation
}

\author{
C. RÉMÉSY, Y. CHILLIARD (*), Y. RAYSSIGUIER, A. MAZUR, C. DEMIGNÉ
}

Laboratoire des Maladies métaboliques,

(*) Laboratoire de la Lactation,

I.N.R.A., Theix, 63122 Ceyrat, France.

Le métabolisme hépatique chez les ruminants a, jusqu'ici, été peu étudié. II existe pourtant une somme importante de connaissance dans ce domaine chez les animaux de laboratoire. Chez les ruminants ces recherches ont progressé depuis peu du fait de l'utilisation de nouvelles techniques telles que les hépatocytes isolés (Clark et al., 1976 ; Ash et Pogson, 1977 ; Pogson et al., 1984). Un des rôles majeurs du foie est de contrôler l'approvisionnement de l'organisme en glucose. De plus, chez les ruminants, la majorité des glucides sont transformés en acides volatils dans le rumen et la synthèse du glucose par le foie joue de ce fait un rôle primordial. Dans ces conditions, la disponibilité en glucose peut être un facteur limitant pour la croissance fœtale, la production laitière ou l'anabolisme corporel.

Lorsque le déséquilibre entre l'apport et l'utilisation potentielle du glucose est très important (gestation, lactation), I'organisme essaie de lutter contre l'hypoglycémie par une mobilisation intense des lipides et des protéines. Une partie des acides gras est métabolisée au niveau du foie via la synthèse des triglycérides ou des corps cétoniques. II existe, en fait, de nombreuses interactions entre néoglucogenèse et cétogenèse que nous développerons en particulier pour décrire les problèmes métaboliques de la brebis gravide ou de la vache en lactation.

\section{Le métabolisme des substrats glucoformateurs}

(fig. 1)

Les principaux substrats glucoformateurs sont le propionate puis les acides aminés et enfin le lactate et le glycérol. Ces substrats entrent dans la voie de la néoglucogenèse au niveau du pyruvate (alanine, lactate, sérine, glycine), d'un intermédiaire du cycle de Krebs (propionate, glutamate, aspartate, proline, etc.) ou des trioses phosphate (glycérol). Certains acides aminés comportent des éléments glucoformateurs et cétoformateurs dans leur chaîne carbonée. La néoglucogenèse est principalement hépatique. Cependant, dans certains cas d'acidose, la néoglucogenèse rénale peut aussi être très active. 


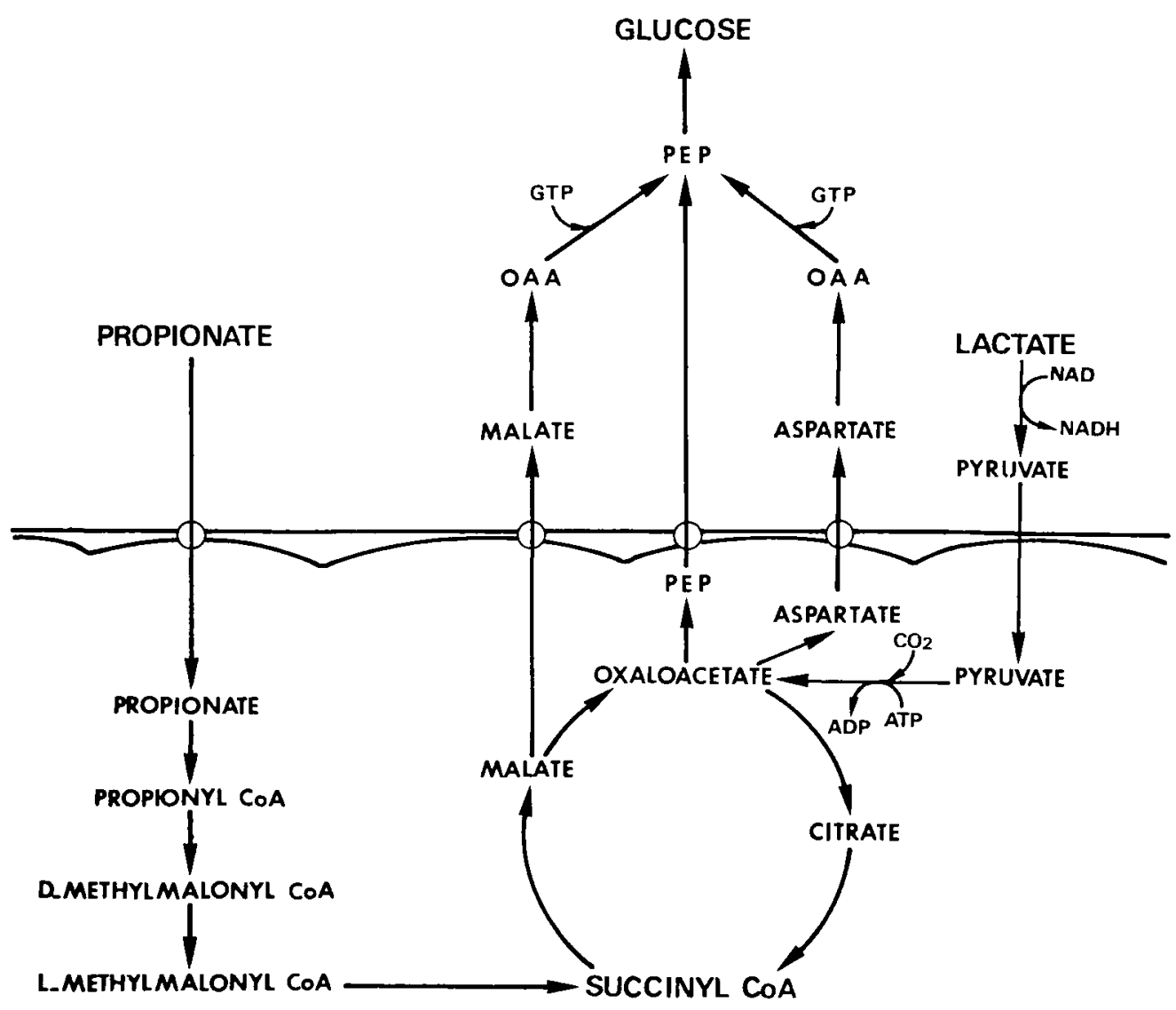

FIG. 1. - Schéma des principales étapes de la néoglucogenèse à partir du lactate ou du propionate.

Chez les monogastriques tels que le rat, la néoglucogenèse hépatique est très finement régulée au niveau de nombreuses enzymes régulatrices, par l'apport digestif en hexoses et par l'état endocrinien. Une surcharge de glucose suffit à inhiber presque entièrement la néoglucogenèse, à condition toutefois que les réponses endocriennes agissent normalement. Par contre, chez les ruminants, les enzymes de la néoglucogenèse hépatique sont toujours très actives à l'état nourri et on peut même observer une diminution de certaines d'entre elles à jeun (Martin et al., 1973). Dans l'ensemble, on note des variations relativement modérées des enzymes de la néoglucogenèse en fonction des situations nutritionnelles ou physiologiques (Mackie et Campbell, 1972.; Mesbah et Baldwin, 1983). Chez les ruminants, l'importance relative des divers substrats glucoformateurs n'est pas connue de façon précise, notamment celle des acides aminés par rapport au propionate. Chez la vache laitière, Wiltrout et Satter (1972) considèrent que le propionate pourrait fournir $50 \%$ du glucose néoformé (au maximum $60 \%$ ) ce qui 
laisse donc une place importante aux autres substrats glucoformateurs. Nous verrons que de nombreux facteurs nutritionnels ou métaboliques peuvent sensiblement modifier ces proportions.

\section{Le métabolisme du propionate.}

Parmi les acides gras volatils, seul le propionate est glucoformateur, l'isobutyrate et le valérate étant peu importants. D'après Bergman et Wolff (1971) plus de $90 \%$ du propionate absorbé est capté par le foie à chaque passage sanguin si bien que des quantités négligeables d'acide propionique sont métabolisées par les tissus périphériques. D'après Baird et al. (1980), le taux d'extraction hépatique varie très peu en fonction de l'état nutritionnel et endocrinien, à la différence des autres composés glucoformateurs. Dans un travail récent (Fafournoux et al., 1985), nous avons étudié le transport du propionate sur hépatocytes isolés de rat et mis en évidence que le propionate passait dans la cellule par un transporteur spécifique qui a une affinité élevée pour le propionate et une forte capacité. Les possibilités d'utilisation hépatique du propionate peuvent cependant être dépassés au-dessus d'un certain seuil de concentration portale $(0,75 \mathrm{mM})$ sans qu'il soit possible d'établir précisément les facteurs limitants : étapes de transport ou métabolisme intracellulaire. Une trop forte absorption peut donc dépasser les capacités d'utilisation hépatique et accroître sa disponibilité à la périphérie.

In vitro, nous avons mis en évidence des différences importantes dans les capacités d'utilisation du propionate et du butyrate par les hépatocytes isolés de rat et de mouton (Demigné et al., 1986a). Alors que chez le rat le butyrate est utilisé environ deux fois plus vite que le propionate, on retrouve un phénomène sensiblement inverse chez les ruminants. Les capacités relativement réduites d'utilisation du butyrate par rapport au propionate sont à mettre en relation avec le fait que le butyrate est fortement métabolisé dans la paroi du rumen.

Dans le foie, le propionate est activé dans la mitochondrie par une propionyl CoA synthétase spécifique (Ricks et Cook, 1981). L'activité de la propionyl CoA carboxylase peut varier en fonction de l'état physiologique et nutritionnel (Baird et Young, 1975). La transformation du méthyl-malonyl CoA en succinyl CoA par une mutase qui a comme cofacteur la vitamine B12, peut constituer une étape limitante dans les cas de carence en cobalt (Peters et Elliot, 1983) et diminuer ainsi la néoglucogenèse à partir du propionate.

Le propionate ne semble pas être entièrement converti en glucose (de 40 à $60 \%$, Young, 1977). Une si faible transformation peut s'expliquer par des interférences au niveau du marquage ; si on applique un facteur de correction de 1,35 pour éliminer les erreurs dues à la dilution du marqueur dans le cycle de Krebs (Wiltrout et Satter, 1972), on aboutit à un rendement supérieur à $60 \%$ (Stangassinger et Giesecke, 1979). In vitro, en comparant le glucose produit au propionate utilisé, nous avons trouvé une transformation d'environ $70 \%$ du propionate en glucose (Demigné et al., 1986b). Les acides gras ont normalement un rôle indispensable pour la fourniture d'énergie et stimulent nettement la néoglucogenèse à partir du lactate chez le rat. Demigné et al. (1986b) n'ont pas trouvé, sur hépatocytes isolés de mouton, des modifications de la néoglucogenèse à partir du pro- 
pionate sous l'influence des acides gras, ou en présence de concentrations élevées en glucose. En fait, les acides gras semblent, dans ce cas, jouer un rôle d'autant moins déterminant que la néoglucogenèse à partir du propionate nécessite peu d'ATP.

\section{Le métabolisme du lactate.}

A la différence du propionate, le lactate peut avoir une origine digestive ou métabolique. Les formes $D$ et $L$ de l'acide lactique ne sont pas toutefois des produits terminaux importants du métabolisme microbien, sauf dans certains cas d'acidose ruminale. L'intestin grêle et la paroi du rumen peuvent produire, à partir du glucose ou du propionate, de l'acide lactique. Compte tenu de leur masse, les territoires musculaires sont les plus importants pour le recyclage du glucose en lactate. Cependant, chez les ruminants, le cycle de Cori est très peu actif (Van der Walt et al., 1983).

Quelle que soit la situation nutritionnelle, le lactate ne fournit qu'une faible part du glucose produit. En début de lactation, lorsqu'il y a une carence en composés glucoformateurs, le foie extrait des proportions plus élevées de lactate (Baird et al., 1980), mais il n'est jamais capable de capter autant de lactate que chez le rat (Rémésy et Demigné, 1983). Les résultats obtenus in vitro (Demigné et al., 1986b) confirment largement la supériorité du propionate sur le lactate comme substrat glucoformateur, sans que les étapes limitantes de l'utilisation du lactate ne soient connues.

L'utilisation hépatique du lactate est souvent limitée par ses faibles concentrations sanguines ; son rôle dans la néoglucogenèse peut être accru en augmentant sa teneur dans le sang (perfusion d'acide lactique) (Naylor et al., 1984). Le lactate s'accumule parfois spontanément dans le sang en cas d'acidose digestive ( $L$ et $\mathrm{D}$ lactate) mais, dans ce cas-là, la baisse du $\mathrm{pH}$ sanguin diminue son utilisation hépatique sans doute au niveau de la pyruvate carboxylase (Cohen et Woods, 1976). Le métabolisme du propionate peut aussi interférer avec l'utilisation du lactate; nous avons montré que la perfusion de propionate à doses élevées bloquait l'utilisation hépatique du lactate et était à l'origine d'hyperlactatémie (Demigné et al., 1986c).

\section{Le métabolisme des acides aminés.}

En cas de déficit en propionate, même s'il existe une utilisation accrue de lactate, il ne s'agit pas d'une formation de glucose à partir de nouvelles chaînes carbonées, seulement d'un recyclage; seule la néoglucogenèse à partir des acides aminés peut compenser le manque de propionate.

Les acides aminés peuvent avoir une origine digestive ou provenir de la mobilisation des protéines corporelles. La protéolyse musculaire se traduit par la libération de certains acides aminés ou aboutit à la production d'alanine et de glutamine. L'alanine peut être utilisée directement par le foie. Les relations interorganes de la glutamine sont complexes puisque cet acide aminé peut être utilisé par les reins s'il y a acidose métabolique (Heitmann et Bergman, 1980). Dans ce der- 
nier cas, le foie peut éliminer le NH3 sous forme de glutamine et pas seulement sous forme d'urée.

Au niveau du foie, l'intensité du catabolisme des acides aminés dépend en premier lieu de leur transport dans la cellule. Chez le rat, de nombreux systèmes de transport concentratifs (dépendant du sodium) ont été caractérisés ; certains sont inductibles par les hormones tels que le système A transportant l'alanine (Fehlmann et al., 1979). Il est probable que des système similaires existent chez les ruminants mais de telles recherches n'ont pas encore été effectuées. Pourtant, le transport revêt sans doute un caractère limitant pour le métabolisme (Rémésy, 1982 ; Fafournoux et al., 1985).

Les acides aminés captés ne sont pas nécessairement transaminés et peuvent servir aux synthèses protéiques. Toutefois, une orientation endocrinienne favorable à la néoglucogenèse (élévation du glucagon et des glucocorticoïdes) se caractérise aussi par une augmentation de la protéolyse hépatique et par une diminution de la protéosynthèse (Hopgood et al., 1980). L'extraction de quatre acides aminés (alanine, glutamine, sérine, glycine) représente à elle seule environ les $2 / 3$ de l'uréogenèse totale (Wolff et al., 1972). Bien que la glycine soit fortement captée par le foie, cet acide aminé à deux atomes de carbone est faiblement néoglucogénique. Par ailleurs, nous avons constaté (Rémésy et Demigné, 1979) qu'il s'accumulait dans certains états pathologiques (brebis cétosiques). Bien que la glutamine puisse être libérée par le foie dans certains cas d'acidose métabolique, cet acide aminé est plus souvent capté par le foie où il est le meilleur substrat de la néoglucogenèse après l'alanine.

Un catabolisme important des acides aminés peut s'accompagner aussi d'une absorption digestive élevée de $\mathrm{NH} 3$ avec les régimes riches en azote. L'uréogenèse étant un processus qui demande beaucoup d'énergie, une forte disponibilité en ammoniaque peut non seulement accroître la lipolyse et le catabolisme des acides gras mais aussi contribuer à diminuer la néoglucogenèse, tout au moins si les résultats in vitro (Demigné et al., 1986a) sont extrapolables in vivo. En revanche, une forte disponibilité en propionate et surtout en butyrate stimulent l'uréogénèse (Demigné et al., 1986a). Chez le rat, des concentrations élevées en propionate inhibent au contraire l'uréogenèse. La synthèse du glucose à partir des acides aminés demande beaucoup plus d'énergie que celle qui a lieu à partir du propionate; la conversion d'alanine en glucose nécessite 10 ATP, celle du propionate 4 ATP seulement. L'utilisation du propionate ou des acides aminés n'aura donc pas les mêmes conséquences énergétiques.

\section{Le métabolisme du glycérol.}

Le glycérol est un excellent substrat pour la néoglucogenèse mais son utilisation hépatique est limitée par ses faibles concentrations sanguines sauf lorsque la lipomobilisation est intense. D'après Bergman (1983), le glycérol pourrait fournir plus de $10 \%$ du glucose. Une contribution aussi élevée est en fait le résultat d'un cycle futile dû à une réestérification importante des acides gras ; dans ces conditions, le tissu adipeux consomme du glucose pour la synthèse du glycérophosphate et libère du glycérol. 
En conclusion, la néoglucogenèse à partir du propionate est primordiale en raison des capacités très élevées du foie pour le transport et le métabolisme de cet anion. Le ruminant a du mal à remplacer le propionate par d'autres substrats glucoformateurs car il n'est pas toujours aisé de faire absorber des quantités suffisantes de glucose ou d'acides aminés dans l'intestin grêle ; de plus, il recycle mal son glucose ; enfin il présente une réponse endocrinienne pour la stimulation de la néoglucogenèse similaire à celle du monogastrique mais de plus faible amplitude (Brockman et Mann, 1974 ; Gow et al., 1981 ; Grizard et al., 1986).

\section{Le métabolisme des lipides}

Chez les ruminants, comme chez les autres espèces, le foie joue un rôle important dans le catabolisme des acides gras ou leur incorporation dans les différentes fractions lipidiques (triglycérides, phospholipides, cholestérol libre ou estérifié).

\section{L'activation des acides gras.}

Le devenir des acides gras et l'efficacité de leur captation dépendent de la longeur de leur chaîne carbonée. Chez les ruminants, l'acétate est peu capté par le foie (Ricks et Cook, 1981) et une grande partie du butyrate est directement métabolisée dans la paroi du rumen.

Le foie métabolise principalement les acides gras longs liés à la fraction albumine (Bell, 1981). Ces acides gras ont pour origine la lipolyse du tissu adipeux et pour une faible part les triglycérides circulants après action de la lipoprotéine lipase. La captation hépatique des acides gras est d'autant plus efficace que le rapport acides gras libres/albumine s'élève. Le foie peut aussi capter directement de faibles quantité de triglycérides (après hydrolyse par une lipase hépatique).

\section{Estérification des acides gras ou $\beta$-oxydation (fig. 2).}

Après leur transfert dans la cellule hépatique, les acides gras libres sont activés en acyl CoA et, à ce stade, il existe un carrefour métabolique qui les dirige soit vers la synthèse des triglycérides ou des phospholipides par l'intermédiaire d'une glycérol phosphate acyl transférase, soit vers l'utilisation mitochondriale ( $\beta$-oxydation) par l'action d'une carnitine acyl transférase I.

La régulation de ce carrefour métabolique a fait l'objet de nombreux travaux chez le monogastrique et peu d'études seulement, à notre connaissance, ont été conduites chez le ruminant (Lomax et al., 1983). Chez le rat, il a été montré que ce n'était pas la disponibilité en glycérol-phosphate, ni l'activité de la glycérolphosphate acyl transférase, qui orientaient le devenir des acyl CoA mais plutôt l'activité de la carnitine acyl transférase I (CATI). Cette enzyme, qui fait pénétrer les acides gras dans la mitochondrie, est justement inhibée par le malonyl CoA, premier intermédiaire de la synthèse des acides gras (Mc Garry et Foster, 1981 ; Zammit, 1981).

Le fait que la lipogenèse de novo soit faible dans le foie du ruminant ne semble pas modifier le rôle du malonyl CoA dans le contrôle de l'oxydation des acides 


\section{Métabolisme mitochondrial des acides gras : oxydation ou cétogenèse.}

Le catabolisme des acides gras dans la mitochondrie dépend exclusivement de l'afflux en acides gras du compartiment cytosolique par le système acyl carnitine que nous venons de décrire puisque la $\beta$-oxydation, qui conduit à la production d'acétyl CoA, est une voie métabolique apparemment peu régulée si ce n'est peut-être par l'état d'oxydo-réduction de la mitochondrie (rapport NADH/NAD). Une forte disponibilité en propionate pourrait toutefois diminuer le catabolisme des acides gras par l'intermédiaire de la séquestration du CoA dans les divers métabolites conduisant au succinyl CoA. Récemment, un autre effet du propionyl CoA sur l'inhibition de l'acyl CoA déshydrogénase a été décrit par Shaw et Engel (1985) et pourrait être responsable des effets inhibiteurs du propionate à concentration physiologique.

Le devenir de l'acétyl CoA loxydation, production de corps cétoniques ou exportation mitochondriale) est l'objet d'un autre carrefour métabolique important. Dans la mesure où les possibilités d'exportation mitochondriale de l'acétyl CoA sont sans doute mineures, la synthèse de citrate (cycle de Krebs) ou la production des corps cétoniques, constituent les deux voies majeures de l'utilisation de l'acétyl CoA. En cas de surproduction d'acétyl CoA, les possibilités de son incorporation dans le cycle de Krebs sont très vite saturées par la disponibilité en oxalo-acétate, voire par l'utilisation de l'énergie produite dans ce cycle. C'est pourquoi la cétogenèse constitue la seule possibilité d'utilisation intensive de l'acétyl CoA lorsque la voie de l'acyl carnitine transférase est très active. Une inhibition de l'activité du cycle de Krebs par un drainage de ces intermédiaires vers la néoglucogenèse accélèrera la cétogenèse. Chez les ruminants, la 3hydroxybutyrate déshydrogénase hépatique est une enzyme dont l'activité est plutôt limitante (Watson et Lindsay, 1972). En cas de très forte cétogenèse, le rapport 3-hydroxybutyrate/acétoacétate a donc tendance à diminuer dans le sang.

La libération d'acétate par le foie lorsque la lipolyse est élevée, peut constituer une autre voie d'utilisation de l'acétyl CoA (Palmquist, 1972; Baird et al., 1975 ; Costa et al., 1976). La production d'acétate endogène a aussi été observée chez des vaches en lactation. Les mécanismes qui orientent l'acétyl CoA vers la production des corps cétoniques ou d'acétate endogène (rôle éventuel de la carnitine) sont mal connus et seule une meilleure connaissance du métabolisme cellulaire permettra de comprendre la signification physiologique de l'acétate endogène et son importance réelle.

En fait, pour qu'un état de cétose se développe, il faut que la production des corps cétoniques dépasse les capacités maximales d'utilisation par les tissus périphériques. Ceci suppose qu'il y ait une mobilisation élevée des acides gras libres, une hypoglycémie et une faible disponibilité en composés glucoformateurs. La simple mobilisation des acides gras n'est pas suffisante pour élever dangereusement la cétogenèse. Un ruminant à jeun, tari et non gravide, peut en effet mobiliser ses lipides sans faire d'acidocétose. 
Synthèse et sécrétion des triglycérides.

Les acides gras qui ne sont pas métabolisés dans la mitochondrie sont principalement estérifiés en triglycérides, phospholipides ou esters de cholestérol. Lorsque l'afflux d'acides gras augmente fortement, il y a surtout une élévation de la synthèse des triglycérides même si la $\beta$-oxydation est nettement accrue dans les cas de cétose. Par ailleurs, il peut exister des situations en début de lactation où la cétogenèse est modérée et la réestérification importante. La synthèse des triglycérides peut alors largement dépasser les possibilités de leur exportation sous forme de lipoprotéines, et il se développe une stéatose (Reid et al., 1979).

Les lipides hépatiques sont normalement sécrétés sous forme de lipoprotéines associant triglycérides, phospholipides, cholestérol et apoprotéines. Classiquement étudiées par ultracentrifugation, les lipoprotéines sont classées selon I'intervalle de densité du milieu dans lequel elles flottent: chylomicrons, VLDL (lipoprotéines de très faible densité), LDL (faible densité), HDL (haute densité). II existerait en réalité une fraction intermédiaire entre les $L D L$ et les HDL dont la signification physiologique est à l'heure actuelle discutée ( $\mathrm{LDL}_{2}$ ou $\mathrm{HDL}_{1}$ ) (Ferreri et Gleockler, 1979 ; Grummer et al., 1983 ; Chapman et Forgez, 1985). Les apoprotéines majeures chez les bovins sont l'Apo $B$ et l'Apo $A_{1}$ pour les VLDL, I'Apo B pour les LDL, l'Apo $A_{1}$ pour les HDL (Forte et al., 1981).

Le ruminant se caractérise par rapport aux autres espèces animales par un taux très faible de chylomicrons et de lipoprotéines légères. Cependant ces classes de lipoprotéines jouent un rôle très important comme source de lipides pour les tissus extra-hépatiques, en particulier pendant la lactation (Ferreri et Elbein, 1982), si bien que le taux extrêmement bas des VLDL chez la vache laitière ne serait pas dû à une synthèse ou une sécrétion hépatique réduites, mais plutôt à une avidité exceptionnelle de la mamelle pour les triglycérides associés à cette fraction (Palmquist et Mattos, 1978 ; Glascock et Welch, 1974 ; Gleockler et al., 1980). La régulation de la sécrétion des lipoprotéines par le foie est peu connue chez le ruminant adulte alors que les travaux réalisés sur foie perfusé ou hépatocytes isolés ont permis d'approfondir ce domaine chez d'autres espèces animales. Une meilleur connaissance de cet aspect est cependant nécessaire à la compréhension des déviations du métabolisme lipidique hépatique, qui sont particulièrement fréquentes chez la vache laitière.

\section{Principales interactions entre le métabolisme des lipides et des glucides au niveau du foie (fig. 3)}

Le métabolisme hépatique chez le ruminant se caractérise par une glycolyse très réduite, une néoglucogenèse élevée et une faible vitesse de synthèse des acides gras (Ballard et al., 1969). Il semble donc exister un antagonisme entre lipogenèse et néoglucogenèse. En effet, il y a une compétition entre ces deux voies métaboliques pour la fourniture d'énergie ou l'utilisation des chaînons carbonés. De plus, il existe plusieurs étapes enzymatiques antagonistes telles que la voie des pentoses, celle de l'enzyme malique et celle de la pyruvate déshydrogénase. 


\section{Les interactions au niveau du devenir du pyruvate.}

Chez les ruminants, les composés glucoformateurs conduisant au pyruvate sont nettement moins bien utilisés vers la néoglucogenèse que ceux dont le métabolisme aboutit à la formation d'un intermédiaire du cycle de Krebs. La pyruvate carboxylase joue un rôle très important chez les ruminants et on observe des variations nettes de son activité en parallèle avec l'intensité de la néoglucogenèse (Filsell et al., 1969 ; Brockman et Mann, 1974 ; Baird et Young, 1975). L'utilisation du pyruvate vers la néoglucogenèse nécessite un catabolisme important des acides gras à plusieurs niveaux : augmentation du transport du pyruvate dans la mitochondrie en échange avec l'acétoacétate, activation de la pyruvate carboxylase et inhibition de la pyruvate déshydrogénase par l'acétyl CoA. Réciproquement, l'utilisation du pyruvate dans la lipogenèse contribue à diminuer la néoglucogenèse lactivation de la pyruvate déshydrogénase, diminution de la $\beta$-oxydation et de la production d'acétyl CoA par I'intermédiaire du malonyl CoA au niveau de la CAT I).

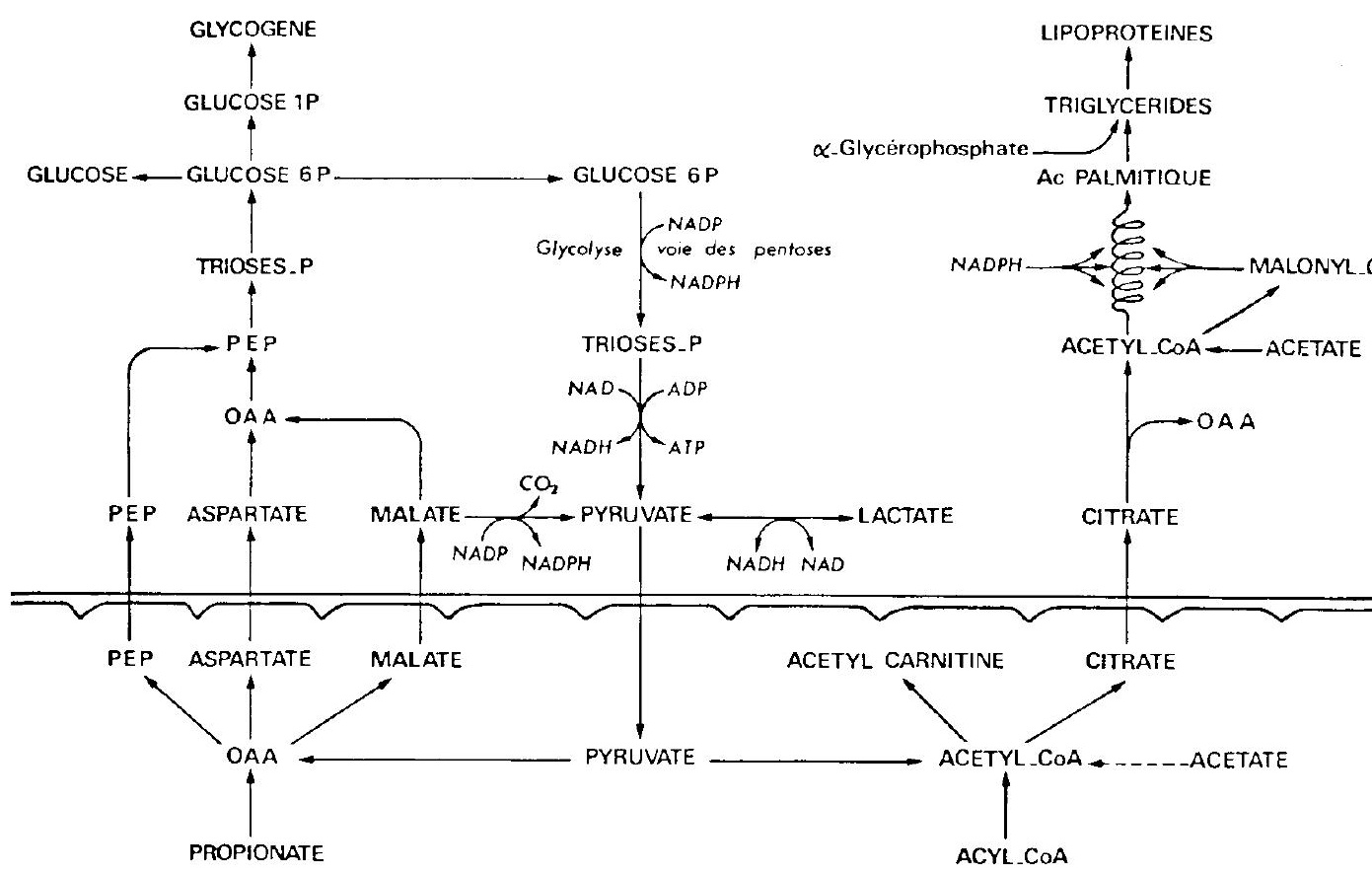

FIG. 3. - Antagonisme entre néoglucogenèse et lipogenèse pour l'utilisation des chaînons carbonés et la fourniture de NADPH.

Les interactions au niveau de l'oxaloacétate.

Chez les ruminants, l'oxaloacétate est essentiellement utilisée vers la néoglucogenèse, d'autant que la phosphoénol-pyruvate carboxykinase est principalement mitochondriale et peut donc être considérée comme une enzyme constitu- 
tive, contrairement au rat. D'ailleurs, un drainage trop important de l'oxoloacétate vers la néoglucogenèse peut diminuer l'activité du cycle de Krebs et stimuler la cétogenèse. Si la lipogenèse était nettement active il pourrait exister d'autres antagonismes avec la néoglucogenèse au niveau du devenir de l'oxaloacétate : inhibition de la sortie mitochondriale du phosphoénol-pyruvate par le citrate (Faulkner et Zammit, 1985), activité de l'enzyme malique et destruction d'une source d'oxaloacétate nécessaire à la synthèse du phosphoénol-pyruvate.

\section{Les interactions au niveau du devenir des trioses-phosphates ou de la glycolyse.}

Les trioses-phosphates provenant du métabolisme des composés glucoformateurs sont très activement convertis en fructose 1,6 diphosphate puisque les enzymes de la néoglucogenèse sont toujours actives chez les ruminants. Les carrefours du fructose 6-phosphate, fructose 1,6 diphosphate et du glucose 6phosphate sont normalement l'objet de "cycles futiles" très importants (flux bidirectionnels entraînant un gaspillage d'ATP) chez les espèces dont le foie peut présenter une glycolyse et/ou une lipogenèse actives. Leur importance est sans doute moindre dans le foie des ruminants qui utilise très peu de glucose mais il est probable que les hormones pancréatiques (insuline et glucagon) modulent l'activité des diverses enzymes-clés (pyruvate kinase, phosphofructokinase) et influencent ainsi la voie de la néoglucogenèse.

La voie des pentoses est peu active dans le foie des ruminants (Mesbah et Baldwin, 1983 ; Chilliard, 1985) ; si cette voie contribuait nettement à la fourniture de NADPH pour la lipogenèse, elle utiliserait une partie du glucose 6-phosphate au détriment de la néoglucogenèse. II peut exister aussi diverses interactions entre lipogenèse et glycolyse par le biais du citrate et du glycéro-phosphate mais l'importance de ces régulations n'est pas connue chez les ruminants (Faulkner et Zammit, 1985). II y a aussi un antagonisme entre lipogenèse et néoglucogenèse pour l'utilisation du glycérol ou du glycérol phosphate vers la synthèse des triglycérides et des phospholipides.

\section{Les interactions au niveau du devenir des acides gras.}

Les composés glucoformateurs, principalement le propionate, stimulent la réestérification des acides gras (Lomax et al., 1983) mais les mécanismes impliqués sont encore inconnus : synthèse du glycérol-phosphate ou inhibition du métabolisme mitochondrial des acides gras longs. II serait intéressant en particulier d'étudier dans quelle mesure le méthyl-malonyl CoA produit dans la mitochondrie, peut agir au niveau cytosolique sur la CAT I à moins qu'il n'agisse directement dans la mitochondrie au niveau de la CAT II.

Lorsqu'il y a pléthore en composés glucoformateurs, la cellule hépatique utilise, dans les diverses voies possibles, y compris la lipogenèse les substrats captés et le catabolisme des acides gras est alors réduit au minimum. II peut y avoir aussi, dans ce cas, une stimulation de la sécrétion des lipoprotéines par une forte sécrétion d'insuline. A l'inverse une forte disponibilité en acyl CoA et acétyl CoA peut, dans une certaine mesure, épargner les composés glucoformateurs mais ceci suppose une inhibition presque totale de la lipogenèse pour permettre une 
entrée suffisante des acyl-carnitines dans les mitochondries. Par conséquent, même si la lipogenèse de novo est faible chez les ruminants, cette voie métabolique exerce un rôle important dans le contrôle du métabolisme cellulaire. Plutôt que l'antagonisme entre lipogenèse et néoglucogenèse, il est préférable de souligner l'importance de la régulation de devenir des acides gras vers l'estérification ou la $\beta$-oxydation et celle du devenir des substrats glucoformateurs plus ou moins transformés en glucose.

En conclusion, le propionate joue un rôle prépondérant dans les interactions entre métabolisme des lipides et des glucides au niveau du foie des ruminants mais ses divers effets doivent encore être précisés : inhibition de l'entrée des acylCoA dans la mitochondrie par le méthylmalony $\operatorname{CoA}$, inhibition de la $\beta$-oxydation par le propionyl CoA, activation du cycle de Krebs...

\section{Le métabolisme hépatique durant la gestation et la lactation}

Durant la gestation et la lactation, les besoins en glucose sont très élevés et l'organisme métabolise de grandes quantités de lipides. Les interactions entre le métabolisme des lipides et des composés glucoformateurs jouent donc un rôle très important, en particulier chez la brebis et la chèvre portant plusieurs fotus, et chez la vache et la chèvre forte productrices en début de lactation.

\section{Mobilisation des réserves.}

L'alimentation ne pouvant couvrir totalement les besoins de l'animal en fin de gestation ou en début de lactation, celui-ci mobilise ses réserves corporelles (tabl. 1). D'un point de vue quantitatif, la mobilisation des lipides est de beaucoup la plus importante (Chilliard et al., 1983 ; Chilliard, 1985). Cette mobilisation dépend à la fois du niveau d'alimentation, du potentiel de production et de l'état des réserves corporelles. En début de lactation, les vaches fortes productrices présentent une diminution de l'insulinémie qui joue certainement un rôle majeur dans la réponse lipolytique observée. En outre, une alimentation excessive avant le vêlage pourrait entraîner, dans un premier temps, de fortes sécrétions d'insuline avec, ultérieurement, l'acquisition d'une résistance périphérique. Ainsi, en début de lactation, coexisteraient de faibles taux $d$ 'insuline et une modification des récepteurs à cette hormone dans les tissus adipeux. En définitive, la lipomobilisation de la vache haute productrice en début de lactation serait favorisée à la fois par une augmentation de la sécrétion d'hormone de croissance, une diminution de l'action de l'insuline et une réponse exagérée aux stress adrénergiques (revues de Bauman et Elliott, 1983 ; Vernon et Flint, 1984 ; Chilliard, 1986). La mobilisation concerne aussi de façon variable mais limitée (généralement moins de $10 \mathrm{~kg}$ ) les protéines corporelles, principalement musculaires (Chilliard et Robelin, 1983 ; tabl. 1). 
TABLEAU 1

Comparaison de l'importance de la mobilisation des réserves chez la brebis et la vache en fin de gestation et en début de lactation.

\begin{tabular}{|c|c|c|}
\hline & $\begin{array}{l}\text { Lipides } \\
(\mathrm{kg})\end{array}$ & $\begin{array}{l}\text { Protéines } \\
\quad(\mathrm{kg})\end{array}$ \\
\hline $\begin{array}{l}\text { Brebis } \\
\quad-\text { fin gestation } \\
\quad \text { (6 sem., } 2-4 \text { fœetus })\end{array}$ & $2-6$ & $0-0,4$ \\
\hline $\begin{array}{l}\text { - début lactation } \\
\text { (6 sem., } 2 \text { agneaux) }\end{array}$ & 0.13 & $0-1,2$ \\
\hline $\begin{array}{l}\text { Vache } \\
\qquad \begin{array}{l}\text { - début lactation } \\
\text { (4 à } 6 \text { sem., } \\
\quad 30-40 \mathrm{~kg} \text { de lait par jour) }\end{array}\end{array}$ & $20-70$ & 2-15 \\
\hline$-\underset{(\mathrm{g} / \mathrm{j})}{\operatorname{mobilisation} / \text { jour }}$ & $200-2500^{*}$ & $0-800$ \\
\hline $\begin{array}{l}\text { Mobilisation maximale } \\
\left(\mathrm{g} / \mathrm{kg}^{0,75} / 6 \mathrm{sem} .\right) \\
\text { - vache } \\
\text { - brebis }\end{array}$ & $\begin{array}{l}600 \\
600\end{array}$ & $\begin{array}{r}120 \\
60\end{array}$ \\
\hline
\end{tabular}

* : dont 20 à $250 \mathrm{~g}$ de glycérol, soit 1 à $10 \%$ du glucose néosynthétisé.

(d'après Chilliard, 1985).

Néoglucogenèse.

Chez les petits ruminants, pour assurer la croissance fœtale qui a lieu principalement durant le dernier mois de gestation, il faut un approvisionnement très important de glucose ou de composés glucoformateurs (Prior et Christenson, 1978 ; Hay et al., 1983). Les besoins en glucose sont, par exemple, de l'ordre de $180 / \mathrm{g}$ jour pour des brebis portant deux fœtus contre $90 \mathrm{~g}$ environ chez des brebis vides. Il est probable que l'organisme maternel épargne du glucose pendant la gestation, puisque les besoins du conceptus sont estimés à $120 \mathrm{~g} /$ jour pour deux fœetus.

Par ailleurs, chez la vache laitière, les besoins d'entretien en glucose sont d'environ $700 \mathrm{~g}$ par jour. On considère que la mamelle utilise environ $60 \mathrm{~g}$ de glucose par litre de lait synthétisé ; il s'ensuit que le besoin en glucose est multiplié plusieurs fois en début de lactation, selon le niveau de la production laitière. II dépasse par exemple $3 \mathrm{~kg}$ par jour chez des vaches produisant plus de $35 \mathrm{~kg}$ de lait (Young, 1977) alors que l'absorption intestinale de glucose est généralement inférieure à 0,5 ou $1 \mathrm{~kg} / \mathrm{j}$.

La fin de la gestation et le début de lactation nécessitent donc une forte néoglucogenèse. Celle-ci s'effectue à partir du propionate, des acides aminés et du lactate (Baird et al., 1980). Si à la suite d'une alimentation insuffisante ou déséquilibrée, et/ou d'une baisse d'appétit, l'apport digestif en propionate devient insuffisant, la néoglucogenèse diminuera et il y aura hypoglycémie. La diminution de la glycémie peut être particulièrement sévère chez les petits ruminants en fin 
de gestation si celle-ci est menée à son terme, par comparaison à l'évolution de la glycémie chez la vache en lactation qui peut plus facilement adapter sa production laitière. II faut noter que le fœtus de ruminant réagit à une baisse de l'approvisionnement en glucose par une utilisation plus importante des acides aminés pour sa propre néoglucogenèse hépatique (Girard et al., 1979) (les fœtus des autres espèces ont une néoglucogenèse hépatique très faible). II existe ainsi une compétition entre le foie maternel et les fœtus pour l'utilisation des acides aminés.

Chez l'animal en lactation, un apport supplémentaire d'acides aminés peut stimuler à la fois la néoglucogenèse hépatique et la protéosynthèse mammaire. II en résulte des accroissements de la captation des autres nutriments par la mamelle, et de l'uréogenèse hépatique. Les besoins énergétiques de l'animal augmente en conséquence, ce qui entraîne une lipomobilisation accrue lorsque l'ingestion est insuffisante (cf. Rémésy et al., 1984).

\section{Cétogenèse.}

Lorsque les besoins en glucose de l'utérus gravide ou de la glande mammaire ne peuvent être satisfaits par les apports digestifs et hépatiques de composés glucoformateurs et de glucose, il se développe des cétoses caractérisées par une hypoglycémie, et une élévation importante des teneurs plasmatiques en 3hydroxybutyrate et en acétoacétate (Kronfeld, 1971 ; Baird, 1977 ; tabl. 2). Cette orientation métabolique est stimulée au niveau hépatique par le rapport glucagon/insuline élevé rencontré dans ces situations physiologiques (Basset, 1978 ; Brockman 1979 ; Gow et al., 1981). En cas de carence relative en composés glucoformateurs, les acyls-CoA passent très facilement dans la mitochondrie, d'autant plus que les concentrations en carnitine sont élevées (Lomax et al., 1983) ; de plus, la surproduction d'acétyl CoA est aggravée par la réduction concomitante de l'activité du cycle de Krebs (faible disponibilité en oxaloacétate). L'élévation des corps cétoniques s'accompagne généralement d'une hypoglycémie, mais celle-ci pourrait toutefois faire place à une glycémie normale ou élevée en cas de forte diminution de la sécrétion d'insuline, ou des réponses à cette hor-

\section{TABLEAU 2}

Comparaison de quelques paramètres sanguins de la néoglucogenèse et de la cétogenèse chez la rate ou la brebis gestantes.

\begin{tabular}{|c|c|c|c|c|}
\hline & \multicolumn{2}{|c|}{ Rate gestante } & \multicolumn{2}{|c|}{ Brebis gestante } \\
\hline & Nourrie & à jeun & Normale & cétosique \\
\hline - Glucose & 4,5 & 3,0 & 2,8 & 1,9 \\
\hline - Alanine & 0,33 & 0,17 & 0,16 & 0,09 \\
\hline - Acides gras libres & 0,6 & 1,8 & 0,5 & 1,2 \\
\hline - Triglycérides & 1,8 & 3,0 & 0,35 & 0,35 \\
\hline - 3-hydroxybutyrate & 0,2 & 2,5 & 0,4 & 2,9 \\
\hline - Acétate & 0,2 & 0,2 & 0,5 & 1,6 \\
\hline
\end{tabular}
1985).

Les résultats $(\mathrm{mM})$ sont les moyennes pour environ 10 animaux (d'après Rémésy et Demigné, 
mone. Cette situation s'accompagnerait également d'une réduction de l'utilisation périphérique des corps cétoniques et de l'acétate (Rémésy et Demigné, 1979).

L'acétonémie en fin de gestation et en début de lactation est donc la conséquence d'un déséquilibre nutritionnel provoquant simultanément une forte lipomobilisation et une carence relative en composés glucoformateurs. Elle survient notamment chez les animaux forts producteurs recevant une ration insuffisante ou présentant des chutes spontanées d'ingestion (cf. Coulon et al., 1984 ; Rémésy et al., 1984). Ce syndrome, ses circonstances d'apparition et son traitement ont fait l'objet de nombreuses revues (cf. Baird, 1982 ; Kronfeld, 1982).

\section{Synthèse et sécrétion des lipides.}

Les acides gras qui ne sont pas métabolisés dans la mitochondrie sont principalement estérifiés en triglycérides, phospholipides et esters de cholestérol. Cependant, le contrôle des productions respectives d'acétate, de corps cétoniques et de triglycérides est à l'heure actuelle peu connu chez le ruminant. Ainsi, la brebis en gestation semblerait moins apte que la rate à capter et à recycler des quantités importantes d'acides gras libres plasmatiques en cas de sousalimentation. En effet, dans cette situation, la triglycéridémie augmente fortement chez la rate, alors que la synthèse et la sécrétion des lipoprotéines serait plus difficile chez la brebis (tabl. 2). Ceci pourrait expliquer l'existence de stéatoses hépatiques en fin de gestation chez la brebis (Smith et Walsh, 1975), alors que ce phénomène est limité chez la rate (Scow et al., 1964 ; Herrera et al., 1969).

L'effet de la lactation sur la synthèse et la sécrétion des lipoprotéines peut s'interpréter de la façon suivante : la lactation nécessite un accroissement de la synthèse et de la sécrétion des triglycérides par le foie, essentiellement dans les lipoprotéines légères, fortement captées par la mamelle (Moore et Christie, 1981). Par rapport au tarissement, la lactation se traduit en effet au niveau plasmatique par une diminution des teneurs en triglycérides, une augmentation de celles du cholestérol et des phospholipides, avec diminution des lipoprotéines légères et augmentation des HDL. Au contraire, le tarissement s'accompagnerait d'une diminution de la sécrétion de lipoprotéines, d'où la diminution du cholestérol et des phospholipides alors que les triglycérides augmentent puisque leur prélèvement est moins important (Raphael et al., 1973 ; Mazur et al., 1986a, b).

\section{Stéatose hépatique.}

Au début de la lactation, le foie doit métaboliser des quantités très élevées d'acides gras libres, mais leur utilisation vers la cétogenèse et la production de $\mathrm{CO}_{2}$ peut être limitée, si bien que l'estérification est très importante. En effet, non seulement le transfert des acides gras libres dans la mitochondrie peut être limitant (système acyl carnitine transférase) mais la $\beta$-oxydation elle-même peut être restreinte par la production d'énergie qui résulte de l'incorporation de l'acétyl CoA dans le cycle de Krebs. L'utilisation de l'acétyl CoA dans la cétogénèse permet d'éviter cet excédent énergétique ; toutefois lorsque la carence en composés glucoformateurs n'est pas très accentuée, l'activité du cycle de Krebs et la production d'énergie sont telles qu'elles réduisent les possibilités de catabolisme 
mitochondrial des acides gras (Bell, 1981). La synthèse des triglycérides demeure donc la seule voie d'utilisation massive des acides gras lorsque la cétogenèse n'est pas maximale.

Comme la sécrétion de triglycérides par le foie n'augmente pas proportionnellement à leur synthèse, il en résulte une infiltration lipidique du foie qui est observée chez toutes les vaches en début de lactation (Reid, 1980). La surface de I'hépatocyte occupée par les gouttelettes lipidiques représente alors environ $20 \%$ de la surface totale. Ces gouttelettes régressent à partir de la $3^{\mathrm{e}}$ semaine de lactation. Cependant, certains animaux peuvent présenter une stéatose sévère qui ne se normalise que lentement. Une classification a été proposée selon laquelle, 1 à 2 semaines après le vêlage, les taux de triglycérides dans le foie inférieurs à $50 \mathrm{mg} / \mathrm{g}$ de poids frais et qui représentent une infiltration lipidique de moins de $20 \%$ sont considérés comme normaux. La stéatose est qualifiée de modérée ou sévère pour des taux de lipides respectivement inférieurs ou supérieurs à $40 \%$ (100 mg/g) (Gaal et al., 1983).

L'accumulation de lipides est essentiellement caractérisée par une augmentation des triglycérides, alors que les taux de cholestérol et de phospholipides sont peu modifiés (Collins et Reid, 1980 ; Mazur et al., 1986c). D'autres changements cytologiques tels que des lésions des mitochondries et une diminution du réticulum endoplasmique ont été décrits et témoignent de graves pertubations du métabolisme de I'hépatocyte quand la stéatose est sévère (Reid et Collins, 1980 ; Reid et al., 1981). Ces lésions mitochondriales pourraient gêner certaines voies métaboliques limitantes telles que la néoglucogenèse. L'accumulation des acyl CoA peut par exemple diminuer de nombreux échanges entre mitochondries et cytoplasme.

\section{Origine et conséquences de la stéatose.}

Chez des vaches ne présentant pas de stéatose grave, le début de lactation, comparé à un stade plus tardif (4e semaine), se caractérise au niveau plasmatique par de faibles teneurs en cholestérol et en phospholipides, en particulier dans la fraction LDL (Mazur et al., 1986a). Chez des vaches fortement stéatosiques, les taux de cholestérol et de phospholipides dans la fraction LDL diminuent en début de lactation, de 40 à $50 \%$ par rapport aux animaux considérés comme normaux (Herdt et al., 1983 ; Rayssiguier et al., 1986b). Il y aurait donc, chez ces animaux, une accentuation de la diminution des lipoprotéines légères qui accompagne normalement le début de la lactation. Ces résultats suggèrent que la stéatose est probablement la conséquence d'une diminution de l'élaboration et/ou de la sécrétion des lipoprotéines légères. Les mécanismes en cause ne sont pas connus et pourraient faire intervenir soit une difficulté de la synthèse des constituants lipidiques autres que les triglycérides, comme le montre la diminution du cholestérol et des phospholipides associés aux LDL, soit une altération de la synthèse des apoprotéines.

L'importance des bases azotées ou des donneurs de méthyl (éthanolamine, choline, bétaïne, méthionine, sérine) pour la synthèse des phospholipides a souvent été soulignée, mais un effet bénéfique éventuel de leur administration n'a pas été montré. Une diminution générale de la synthèse protéique, et plus parti- 
culièrement des apoprotéines, à la suite d'une stimulation de la protéolyse et d'une réduction du réticulum endoplasmique pourrait également être invoquée.

La stéatose s'accompagne d'une fréquence accrue de diverses maladies métaboliques (acétonémie en particulier, Groehn et al., 1983), qui ont fait l'objet de plusieurs revues (Rayssiguier et al,, 1981 ; Reid et Roberts, 1983 ; Rémésy et al., 1984).

L'observation d'un plus grand nombre de métrites et de mammites chez les vaches stéatosiques a conduit à I'hypothèse d'une défaillance de l'immunité cellulaire. Mais les travaux actuels ne permettent cependant pas de savoir si les déviations du métabolisme lipidique qui caractérisent le début de la lactation sont directement la cause d'une susceptibilité accrue à l'infection. Une diminution de la fertilité a également été observée elle est attribuée à un retard de l'activité ovarienne et à une diminution du taux de réussite des inséminations (Reid, 1983).

Une infiltration lipidique modérée du foie accompagne normalement le début de la lactation. Ce phénomène réversible peut être considéré comme physiologique (Reid, 1980). Cependant en raison de la fréquence des accidents du postpartum lors de stéatose excessive, l'intérêt des recherches dans ce domaine s'est considérablement accrue ces dernières années (Revue de Reid et Roberts, 1983).

Plusieurs expérimentations ont souligné l'importance de l'état corporel au moment du vêlage sur l'apparition de la stéatose hépatique en début de lactation, les animaux gras présentant un risque accru par suite d'une lipomobilisation plus intense (Fronk et al., 1980 ; Treacher et al., 1981; Rayssiguier et al., 1986a ; revue de Fromageot et al., 1980).

II faut toutefois souligner ici que ce serait l'amaigrissement excessif en début de lactation, et non un état d'engraissement élevé au vêlage, qui serait le principal facteur de risque de troubles métaboliques et de l'appareil génital, et de retards dans la reproduction (selon l'enquête de l'EDE Bretagne - Pays de Loire sur 3500 vaches dont $43 \%$ étaient grasses ou très grasses ; Disenhaus et al., 1985). L'association entre troubles sanitaires et stéatose hépatique proviendrait donc essentiellement d'un effet néfaste d'une forte lipomobilisation en début de lactation, soulignant le danger de toute sous-alimentation excessive des vaches grasses.

En conclusion, les besoins en glucose chez les ruminants durant la gestation ou la lactation sont très importants et la néoglucogenèse peut, dans ces conditions, devenir une fonction limitante. Les difficultés d'approvisionnement en glucose ou en composés glucoformateurs accroissent en retour la mobilisation des lipides ou des protéines corporels, ce qui peut avoir des conséquences pathologiques graves. De nombreux points doivent être approfondis pour comprendre les diverses relations qui existent entre le métabolisme des glucides, des protéines et des lipides. Les concepts actuels dans ce domaine proviennent en partie d'extrapolations de résultats obtenus chez les animaux de laboratoire. Compte tenu des particularités du métabolisme des ruminants, l'avancée des recherches dans ce domaine nécessitera, en plus des études in vivo effectuées jusqu'ici, I'utilisation des modèles appropriés in vitro, tels que les hépatocytes isolés. 


\section{Références}

ASH R., POGSON C. I., 1977. Preparation and biochemical characterization of isolated parenchymal cells from adult sheep liver. Biochim. biophys. Acta., 496, 475-483.

BAIRD G. D., 1977. Aspects of ruminant intermediary metabolism in relation to ketosis. Biochem. Soc. Trans., 5, 819-827.

BAIRD G. D., 1982. Primary ketosis in the high-producing dairy cow: clinical and subclinical disorders, treatement, prevention, and outlook. J. Dairy Sci., 65, 1-10.

BAIRD G. D., LOMAX M. A., SYMONDS H. W., SHAW S. R., 1980. Net hepatic and splanchnic metabolism of lactate, pyruvate and propionate in dairy cows in vivo in relation to lactation and nutrient supply. Biochem. J., 186, 47-57.

BAIRD G. D., SYMONDS H. W., ASH R., 1975. Some observations of metabolite production and utilization in vivo by the gut and liver of adult dairy cows. J. agric. Sci., 85, 281-296.

BAIRD G. D., YOUNG J. L., 1975. The response of key gluconeogenic enzymes in bovine liver to various dietary and hormonal regimes. J. agric. Sci., Camb., 84, 227-230.

BALLARD F. J., HANSON R. W., KRONFELD D. S., 1969. Gluconeogenesis and lipogenesis in tissue from ruminant and non ruminant animals. Fed. Proc., 28, 218-230.

BASSET J. M., 1978. Endocrine factors in the control of nutrient utilization : ruminants. Proc. Nutr. Soc., 37, 273-279.

BAUMAN D. E., ELLIOT J. M., 1983. Control of nutrient partitioning in lactating ruminants. 437-468. In : MEPHAM T. B., Biochemistry of lactation, Elsevier.

BELL A. W., 1981. Lipid metabolism in liver and selected tissues and in the whole body of ruminant animals. In : CHRISTIE W. W., Lipid metabolism in ruminant animals. Pergamon Press., 363410.

BERGMAN E. N., 1983. The pools of cellular nutrients : glucose. In : RUIS P. M., World animal science, dynamic biochemistry of animal production. Elsevier, 9, p. 173.

BERGMAN E. N., WOLFF J. E., 1971. Metabolism of volatile fatty acids by liver and portaldrained viscera in sheep. Am. J. Physiol., 221, 586-592.

BRINDLE N. P. J., ZAMMIT V. A., POGSON C. 1., 1985. Inhibition of sheep liver carnitine palmitoyltransferase by methylmalonyl CoA. Biochem. Soc. Trans., 13, 880-881.

BROCKMAN R. P., 1979. Roles for insulin and glucagon in the development of ruminant ketosis a review. Can. vet. J., 20, 121-126.

BROCKMAN R. P., MANNS J. G., 1974. Effects of glucagon on activities of hepatic enzymes in sheep. Cornell Vet., 64, 217-224.

CHAPMAN M. J., FORGEZ P., 1985. Lipid transport systems : some recent aspects in swine, cattle and trout during development. Reprod. Nutr. Dévelop., 25, 217-226.

CHILLIARD Y., 1985. Métabolisme du tissu adipeux, lipogenèse mammaire et activités lipoprotéineslipasiques chez la chèvre au cours du cycle gestation-lactation. Th. Doct. Etat ès Sci., Univ. $\mathrm{P}$ et $\mathrm{M}$. Curie, Paris VI.

CHILLIARD Y., 1986. Revue bibliographique: Variations quantitatives et métabolisme des lipides dans les tissus adipeux et le foie au cours du cycle gestation-lactation chez la ratte, la brebis et la vache. Reprod. Nutr. Dévelop., (sous presse).

CHILLIARD Y., REEMOND B., SAUVANT D., VERMOREL M., 1983. Particularités du métabolisme énergétique des hautes productrices de lait. Bull. Tech. CRZV Theix, I.N.R.A., 53, 37-64.

CHILLIARD Y., ROBELIN J., 1983. Protein mobilization in early lactating cows measured by slaughter and $\mathrm{D}_{2} \mathrm{O}$. dilution techniques. 4ème Symp. int. Métabol. Nutr. Azoté. ClermontFd, EAAP Publ. $n^{\circ} 31$, vol. II, 195-198.

CLARK M. G., FILSELL O. H., JARRETT I. G., 1976. Gluconeogenesis in isolated intact lamb liver cells. Biochem. J., 156, 671-680.

COHEN R. D., WOODS H. F., 1976. In COHEN R. D., WOODS H. F. Clinical and biochemical aspects of lactic acidosis. Blackwell, Londres, pp. 123-161.

COLLINS R. A., REID I. M., 1980. A correlated biochemical and stereological study of periparturient fatty liver in the dairy cow. Res. vet. Sci., 28, 373-376.

COSTA N. D., Mc INTOSH G. H., SNOSWELL A. M., 1976. Production of endogenous acetate by the liver in lactating ewes. Austr. J. biol. Sci. 29, 33-42. 
COULON J. B., DOREAU M., REMOND B., JOURNET M., 1984. Capacité d'ingestion des vaches laitières en début de lactation. I. Evolution, liaison avec certains paramètres zootechniques et conséquences sur la production laitière. 2. Les anomalies de l'ingestion : liaisons avec l'état sanitaire et quelques paramètres du métabolisme énergétique et de la digestion. Bull. tech. C.R.Z.V. Theix, I.N.R.A., 55, 33-51.

DEMIGNE C., YACOUB C., RÉMÉSY C., FAFOURNOUX P., 1986a. Propionate and butyrate metabolism in rat or sheep hepatocytes. Biochim. biophys. Acta (sous presse).

DEMIGNE C., FARFOURNOUX P., REMEESY C., YACOUB C., 1986b. Utilisation des substrats glucoformateurs par les hépatocytes isolés de mouton. Reprod. Nutr. Dévelop., 26, 369-370.

DEMIGNE C., YACOUB C., REMESY C., 1986c. Comparaison des effets glucogéniques et anticétogéniques du propionate, du propylène glycol et du glucose chez le mouton. Reprod. Nutr. Dévelop., 26, 367-368.

DISENHAUS C., AUGEARD P., BAZIN S., 1985. Nous, les vaches taries. Influence de l'alimentation pendant le tarissement sur la santé, la reproduction et la production en début de lactation. Résultats d'une enquête de 3 ans sur 3500 V.L. Doc. E.D.E. Bretagne-Pays de Loire (65 p.).

FAFOURNOUX P., RÉMÉSY C., DEMIGNÉ C., 1985. Propionate transport in rat liver cells. Biochim. biophys. Acta, 818, 73-80.

FAULKNER A., ZAMMIT V. A., 1985. Carbohydrate and lipid interrelationships in the liver. Hannah Res., 121-128. Report 1984.

FEHLMANN M., LE CAM A., FREYCHET P., 1979. Insulin and glucagon stimulation of amino acid transport in isolated Rat hepatocytes : synthesis of a high affinity component of transport. $J$. biol. Chem., 254, 10431-10437.

FERRERI L. F., GLEOCKLER D. M., 1979. Electrophoretic characterization of bovine lipoprotein subfractions isolated by agarose gel chromatography. J. Dairy Sci., 62, 1577-1582.

FERRERI L. F., ELBEIN R. C., 1982. Fractionation of plasma triglyceride-rich lipoproteins of the dairy cow : evidence of chylomicron-size particles. J. Dairy Sci., 65, 1912-1920.

FILSELL O. H., JARRETT I. G., TAYLOR P. H., KEECH B., 1969. Effects of fasting, diabete and glucocorticoids on gluconeogenic enzymes in the sheep. Biochim. biophys. Acta, 184, 54.

FORTE T. M., BELL-QUINT J. J., CHENG F., 1981. Lipoproteins of fetal and newborn calves and adult steer: A study of developmental changes. Lipids, 16, 240-245.

FROMAGEOT D., SOYEUX Y., SAVEY M., 1980. Causes de la stéatose. Facteurs alimentaires et zootechniques. In : Le syndrome de la vache grasse. Société française de Buiatrie, 101-176.

FRONK T. J., SCHULTZ L. H., HARDIE A. R., 1980. Effect of dry period overconditionning on subsequent metabolic disorders and performance of dairy cows. J. Dairy Sci., 63, 10801090.

GAAL T., REID I. M., COLLINS R. A., ROBERTS C. J., PIKE B. V., 1983. Comparison of biochemical and histological methods of estimating fat content of liver of dairy cows. Res. vet. Sci, 34, 245-248.

GIRARD J., PINTADO E., FERRE P., 1979. Fuel metabolism in the mammalian fetus. Ann. Biol. anim. Bioch. Biophys., 19, 181-197.

GLASCOCK R. F., WELCH V. A., 1974. Contribution of the fatty acids of three low density serum lipoprotein to bovine milk fat. J. Dairy Sci, 57, 1364-1370.

GLEOCKLER D. M., FERRERI L. F., FLAIM E., 1980. Lipoproteins patterns in normal lactating cows bled at various times : effects of milking Proc. Soc. exp. Biol. Med., 165, 118-122.

GOW C. B., McDOWELL G. H., ANNISON E. F., 1981. Control of gluconeogenesis in the lactating sheep. Aust. J. biol. Sci., 34, 469-478.

GRIZARD J., BALAGE M., MANIN M., 1986. Contrôle hormonal du métabolisme hépatique chez les ruminants. Reprod. Nutr. Dévelop., 26, 245-257

GRUMMER R. R., DAVIS C. L., HEGARTY H. M., 1983. Comparison of ultracentrifugation and gel filtration for the isolation of bovine lipoproteins. Lipids, 18, 795-802.

GROEHN Y., LINDBERG L. A., BRUSS M. L., FARVER T. B., 1983. Fatty infiltration of liver in spontaneously ketotic dairy cows. J. Dairy Sci, 66, 2320-2328.

HAY W. W. Jr., SPARKS J. W., WILKENING R. B., BATTAGLIA F. C., MESCHIA G., 1983. Partition of maternal glucose between conceptus and maternal tissues in sheep. Am. J. Physiol., 245, E347-E350. 
HEITMAN R. N., BERGMAN E. N., 1980. Integration of amino acid metabolism in sheep : effects of fasting and acidosis. Am. J. Physiol., 239, E248-E254.

HENDERSON G. D., XUE G. P., SNOSWELL A. M., 1983. Carnitine and creatine content of tissues of normal and alloxan-diabetic sheep and rats. Comp. Biochem. Physiol., 76B, 295-298.

HERDT T. H., LIESMAN J. S., GERLOFF B. J., EMERY R. S., 1983. Reduction of serum triacylglycerol-rich lipoprotein concentrations in cows with hepatic lipidosis. Am. J. vet. Res., 44, 293-296.

HERRERA E., KNOPP R. H., FREINKEL N., 1969. Carbohydrate metabolism in pregnancy. VI. Plasma fuels, insulin, liver composition, gluconeogenesis and nitrogen metabolism during late gestation in the fed and fasted rat. J. clin. Invest., 48, 2260-2272.

HOPGOOD M. F., CLARK M. G., BAUARD F. J., 1980 . Protein degradation in hepatocyte monolayers. Effects of glucagon, adenosine $3^{\prime}-5^{\prime}$ cyclic monophosphate and insulin. Biochem. J., 186, 71-76.

KATZ J., McGARRY J. D., 1984. The glucose paradox : is glucose a substrate for liver metabolism? J. clin. Invest., 74, 1901-1909.

KRONFELD D. S., 1971. Hypoglycemia in ketotic cows. J. Dairy Sci, 54, 949-961.

KRONFELD D. S., 1982. Major metabolic determinants of milk volume, mammary efficiency and spontaneous ketosis in dairy cows. J. Dairy Sci., 65, 2204-2212.

LOMAX M. A., DONALDSON I. A., POGSON C. I., 1983. The control of fatty acid metabolism in liver cells from fed and starved sheep. Biochem. J., 214, 553-560.

Mac GARRY J. D., FOSTER D. W., 1981. Importance of experimental conditions in evaluating the malonyl COA sensitivity of liver carnitine acyltransferase. Biochem. J., 200, 217-223.

MACKIE W. S., CAMPBELL R. M., 1972. Effects of pregnancy and lactation on the activities of some gluconeogenic and urea-cycle enzymes in sheep liver. J. agr. Sci. Camb., 79, 423-429.

MARTIN R. J., WILSON L. L., COWAN R. L., SINK J. D., 1973. Effects of fasting and diet on enzyme profiles in ovine liver and adipose tissue. J. anim. Sci, 36, 101-106.

MAZUR A., GUEUX E., CHILLIARD Y., RAYSSIGUIER Y., 1986a. Evolution des lipides et lipoprotéines plasmatiques chez la vaches en début de lactation. Reprod. Nutr. Dévelop., 26, 357-358.

MAZUR A., GUEUX E., BAZIN S., RAYSSIGUIER Y., 1986b. Evolution du cholestérol lié aux lipoprotéines légères chez la vache en début de lactation et relation avec l'infiltration lipidique du foie. Reprod. Nutr. Dévelop., 26, 363-364.

MAZUR A., BAUCHART D., CHILLIARD Y., DIDIER R., RAYSSIGUIER Y., 1986c. Teneur en lipides du foie chez la vache en début de lactation: comparaison de différentes techniques d'estimation des triglycérides hépatiques. Reprod. Nutr. Dévelop., 26, 355-356.

MESBAH M. M., BALDWIN R. L., 1983. Effects of diet, pregnancy and lactation on enzyme activity and gluconeogenesis in ruminant liver. J. Dairy Sci, 66, 783-788.

MOORE J. H., CHRISTIE W. W., 1981. Lipid metabolism in the mammary gland of ruminant animals, 227-277. In CHRISTIE W. W., Lipid metabolism in ruminant animals. Pergamon Press.

NAYLOR J. M., KRONFELD D. S., FREEMAN D. E., RICHARDSON D., 1984. Hepatic and extrahepatic lactate metabolism in sheep : effects of lactate loading and pH. Am. J. Physiol., 247, E747-E755.

PALMQUIST D. L., 1972. Palmitic acid as a source of endogenous acetate and hydroxybutyrate in fed and fasted ruminants. J. Nutr., 102, 1401-1411.

PALMQUIST D. L., MATTOS W., 1978. Turnover of lipoproteins and transfer to milk fat of dietary (1-carbon-14) linoleic acid in lactating cows. J. Dairy Sci., 61, 561-565.

PETERS J. P., ELLIOT J. M., 1983. Effect of vitamin B12 status on performance of the lactating ewe and gluconeogenesis from propionate. J. Dairy Sci, 66, 1917-1925.

POGSON C. I., CARPENTIER W. R., COOK J. S., FISHER M. J., LOMAX M. A., SALTER M., STANLEY J. C., 1984. A critical approach to the use of isolated liver cells for the study of metabolic events. Proc. Nutr. Soc., 43, 119-132.

PRIOR R. L., CHRISTENSON R. K., 1978. Insulin and glucose effects on glucose metabolism in pregnant and nonpregnant ewes. J. anim. Sci., 46, 201-210.

RAPHAEL B. C., DIMICK P. S., PUPPIONE D. L., 1973. Lipid characterization of bovine lipoproteins throughout gestation and lactation. J. Dairy Sci., 56, 1025-1032. 
RAYSSIGUIER Y., RÉMÉSY C., DEMIGNÉ C., 1981. Milieu et maladies métaboliques. In : Milieu, Pathologie et prévention chez le Ruminant. I.N.R.A., Publ. ed., Versailles, pp. 175-183.

RAYSSIGUIER Y., MAZUR A., REMOND B., CHILLIARD Y., GUEUX E., 1986a. Influence de l'état corporel au vélage et du niveau d'alimentation en début de lactation sur la stéatose hépatique chez la vache laitière. Reprod. Nutr. Dévelop. 26, 359-360.

RAYSSIGUIER Y., MAZUR A., REID I. M., ROBERTS C. J., GUEUX E., 1986b. Modification des lipoprotéines plasmatiques associées à la stéatose hépatique chez la vache en début de lactation. Reprod. Nutr. Dévelop., 26, 361-362.

REID I. M., COLLINS R. A., BAIRD G. D., ROBERTS C. J., SYMONDS H. W., 1979. Lipid production rates and the pathogenesis of fatty liver in fasted cows. J. agric. Sci, 93, 253-256.

REID I. M., 1980. Incidence and severity of fatty liver in dairy cows. Vet. Rec., 107, 281-284.

REID I. M., COLLINS R. A., 1980. The pathology of postparturient fatty liver in high-yielding dairy cows. Invest. Cell Pathol., 3, 237-249.

REID I. M., COLLINS R. A., PATTERSON A., TREACHER R. J., 1981. Organelle changes in the liver cells of dairy cows around the time of calving. J. comp. Path., 190, 245-250.

REID I. M., DEW S. M., COLLINS R. A., DUCKER M. J., BLOOMFIELD G. A., MORANT S. V., 1983. The relationship between fatty liver and fertility in dairy cows : a farm investigation. J. agric. Sci. Camb., 101, 499-502.

REID I. M., ROBERTS C. J., 1983. Subclinical fatty liver in dairy cows. Current research and futur prospects. Irish Vet. J., 37, 104-110.

REID J. C. W., HUSBANDS D. R., 1985. Oxidative metabolism of long chain fatty acids in mitochondria from sheep and rat liver. Biochem. J., 225, 233-237.

RÉMÉSY C., 1982. Influence de la disponibilité en glucides et en composés glucoformateurs sur le métabolisme intestinal et hépatique chez le rat. Th. Doct. ès Sci., E310, Univ. Clermont II, $116 \mathrm{p}$.

REMESY C., CHILLIARD Y., AROEIRA L., MAZUR A., FAFOURNOUX P., DEMIGNÉ C., 1984. Le métabolisme des lipides et ses déviations chez le Ruminant durant la gestation et la lactation. Bull. Tech. C.R.Z.V. Theix I.N.R.A., 55, 53-71.

RÉMÉSY C., DEMIGNÉ C., 1979. Effects of a reduction in food intake during late pregnancy on gluconeogenesis and ketogenesis in twin pregnant ewes. Ann. Biol. anim. Biochim. Biophys., 19, 141-150.

RÉMÉSY C., DEMIGNE C., 1983. Changes in availability of glucogenic and ketogenic substrates and liver metabolism in fed or starved rats. J. Nutr. Metabol., 27, 57-70.

RÉMÉSY C., DEMIGNÉ C., 1985. Adaptation of hepatic gluconeogenesis and ketogenesis to altered supply of substrates during late pregnancy in the rat. J. Develop. Physiol. (sous pressel.

RICKS C. A., C.OOK R. M., 1981. Regulation of volatile fatty acid uptake by mitochondrial acyl CoA synthetases of bovine liver. J. Dairy Sci., 64, 2324-2335.

SCOW R. O., CHERNICK S. S., BINLEY M. S., 1964. Hyperlipemia and ketosis in the pregnant rat. Am. J. Physiol., 206, 796-804.

SHAW L., ENGEL P. C., 1985. The suicide inactivation of ox liver short-chain acyl-CoA dehydrogenase by propionyl-CoA. Biochem. J., 230, 723-731.

SMITH R. W., WALSH A., 1975. The composition of the liver lipids of the ewe during pregnancy and lactation. Res, vet. Sci., 19, 230-232.

STANGASSINGER M., GIESECKE D., 1979. Quantitative measurement of gluconeogenesis from isobutyrate in sheep. Arch. int. Physiol. Bioch., 87, 265-274.

TILGHMAN S. M., HANSON R. W., BALLARD F. J., 1976. Hormonal regulation of phosphoenolpyruvate carboxykinase (GTP) in mammalian tissues. In HANSON R. W., MEHLMAN M. A. Gluconeogenesis : its regulation in mammalian species. Wyley Intersci., New York, pp. 4791.

TREACHER R. J., REID I. M., ROBERTS C. J., 1981. The effect of body condition at calving on the development of fatty liver and metabolic disease. Anim. Prod., 32, 362. (Abstr.).

VAN DER WALT J. G., BAIRD G. D., BERGMAN E. N., 1983. Tissue glucose and lactate metabolism and interconversions in pregnant and lactating sheep. Brit. J. Nutr., 50, 267-280.

VERNON R. G., FLINT D. J., 1984. Adipose tissue : metabolic adaptation during lactation. Symp. Zool. Soc. Lond., 51, 119-145. 
WATSON H. R., LINDSAY D. B., 1972. 3-hydroxybutyrate dehydrogenase in tissues from normal and ketonaemic sheep. Biochem. J., 128, 53-57.

WEEKES T. E. C., 1974. The " in vitro " metabolism of propionate and glucose by the rumen epithelium. Comp. Biochem. Physiol., 4913, 393-406.

WILTROUT D. W., SATTER L. D., 1972. Contribution of propionate to glucose synthesis in the lactating and non lactating cow. J. Dairy Sci., 55, 307-317.

WOLFF J. E., BERGMAN E. N., WILLIAMS H. H., 1972. Net metabolism of plasma amino acids by liver and portal drained viscera of fed sheep. Am. J. Physiol., 223, 438-446.

YOUNG J.W., 1977. Gluconeogenesis in cattle : significance and methodology. J. Dairy Sci., 60, 115.

ZAMMIT V. A., 1981. Regulation of hepatic fatty acid metabolism. Biochem. J., 198, 75-83. 